TITLE:

\title{
Identification of glucosylceramides containing sphingatrienine in maize and rice using ion trap mass spectrometry.
}

\section{$\operatorname{AUTHOR}(\mathrm{S}):$}

Sugawara, Tatsuya; Duan, Jingjing; Aida, Kazuhiko; Tsuduki, Tsuyoshi; Hirata, Takashi

\section{CITATION:}

Sugawara, Tatsuya ...[et al]. Identification of glucosylceramides containing

sphingatrienine in maize and rice using ion trap mass spectrometry.. Lipids 2010, 45(5): 451-455

\section{ISSUE DATE:}

2010-05

URL:

http://hdl.handle.net/2433/126699

\section{RIGHT:}

The original publication is available at www.springerlink.com; This is not the published version. Please cite only the published version.; この 論文は出版社版でありません。引用の際には出版社版をご確認ご利用 ください。 
1

2

3

4 Tatsuya Sugawara ${ }^{1 *}$, Jingjing Duan $^{1}$, Kazuhiko Aida $^{2}$, Tsuyoshi Tsuduki $^{3}$ and Takashi Hirata ${ }^{1}$

5

6

\section{Identification of Glucosylceramides Containing Sphingatrienine in Maize and Rice using Ion Trap Mass Spectrometry}

\footnotetext{
${ }^{1}$ Division of Applied Biosciences, Graduate School of Agriculture, Kyoto University, Kyoto 606-8502, Japan ${ }^{2}$ Central Laboratory, Nippon Flour Mill Company Ltd., Kanagawa 243-0041, Japan

${ }^{3}$ Laboratory of Food and Biomolecular Science, Graduate School of Life Science and Agriculture, Tohoku University, Sendai 981-8555, Japan
} 1

Running Head: Glucosylceramides containing sphingatrienine in maize and rice

.

*Correspondence to:

Tatsuya Sugawara

Graduate School of Agriculture

Kyoto University

Kyoto 606-8502, Japan

E-mail: sugawara@ kais.kyoto-u.ac.jp

20 
1 Abstract We characterized the glucosylceramide moieties from maize and rice using liquid

2 chromatography-ion trap mass spectrometry. Glucosylceramides containing 4,8-sphingadienine

3 (d18:2) acylated to hydroxy-fatty acids were detected as the predominant molecules both in

4 maize and in rice. In addition, 4-hydroxy-8-sphingenine (t18:1) and sphingatrienine (d18:3)

5 were found in maize and rice glucosylceramides, and in the case of rice, sphingenine (d18:1) was

6 also detected. Glucosylceramides containing d18:3 were acylated to hydroxyl fatty acids (16 to

724 carbon atoms). Our results indicate the presence of the triene type of sphingoid base in

8 higher plants.

9

10 Abbreviations

11 d18:0 Sphinganine

12 d18:1 Sphingenine

13 d18:2 Sphingadienine

14 d18:3 Sphingatrienine

15 t18:0 Phytosphingosine

16 t18:1 Hydoroxysphingeine

17 HPLC High-performance liquid chromatography

18 MS mass spectrometry

Introduction

21 Sphingolipids are found in a wide variety of organisms, and constitute a family of compounds

22 that have a sphingoid base (long-chain base) with an amide-linked fatty acid and a polar head

23 group. The hydrolyzed products of sphingolipids (ceramides and sphingoid bases) are highly 
bioactive compounds that play roles as second messengers that are known to be involved in many aspects of cell regulation, such as cell growth, cell differentiation and apoptosis [1-3]. Recently, dietary sphingolipids have gained attention for their potential to protect the intestine from inflammation and cancer [4-9]. In addition, other physiological functions of sphingolipids, such as improving the barrier function of skin, lowering plasma lipids and prevention of melanin

6 formation, have also been reported [10-12].

Diverse structures of the sphingoid base occur in nature. The most common sphingoid base of mammalian sphingolipids is sphingosine (trans-4-sphingenine, d18:14). Smaller amounts of other sphingoid bases, such as sphinganine (dihydrosphingosine, d18:0) and phytosphingosine (4-hydroxysphinganine, t18:0) are frequently encountered. The structures of

11 sphingoid bases in higher plants are more complicated than in mammals [13]. Plants primarily contain cis- and trans- isomers of $\Delta 8$-unsaturated sphingoid bases, such as 8-sphingenine understand the functional and nutritional significance of dietary sphingolipids. chemical structures of lipids including sphingolipids [14-16]. In this study, we characterized the structures of glucosylceramide, one of the predominant glycosphingolipids in plants, from

19 rice and from maize using liquid chromatography-ion trap mass spectrometry. Our results demonstarate the presence of sphingatrienine (d18:3) in higher plants, which has been described

21 previously in marine invertebrates [17-19] and was found in tobacco leaf [20]. 
Materials

Glucosylceramides were prepared from maize grain and from rice grain using a silica gel column after lipid extraction and saponification as described previously [21]. All other chemicals and

4 solvents were of reagent grade. An HPLC system coupled to LCMS-IT-TOF equipped with an electrospray ionization interface

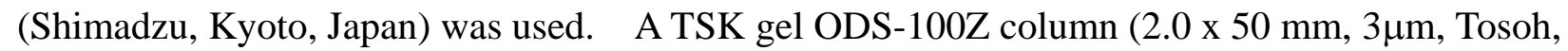
9 Tokyo, Japan) was eluted with acetonitrile/water $(93: 7, \mathrm{v} / \mathrm{v})$ at a flow rate of $0.2 \mathrm{~mL} / \mathrm{min}$. The MS was operated with the following conditions: probe voltage of $4.50 \mathrm{kV}$, CDL temperature of $11200^{\circ} \mathrm{C}$, block heater temperature of $200^{\circ} \mathrm{C}$, nebulizer gas flow of $1.5 \mathrm{~L} / \mathrm{min}$, ion accumulation 12 time of $100 \mathrm{msec}$, MS range of $\mathrm{m} / \mathrm{z} 650$ to $900, \mathrm{MS}^{2}$ range of $\mathrm{m} / \mathrm{z} 200$ to 300 , and CID 13 parameters as follows: energy, 60\%; collision gas 60\%. For the structural analysis of 14 glucosylceramide, $[\mathrm{M}+\mathrm{H}-18]^{+}$(loss of water) in the positive scan mode was used for MS/MS analysis to obtain the product ions. The typical signals which are characteristic for the sphingoid base moieties were observed as the product ions using the auto MS/MS detection

17 mode. In this system, product ions corresponding to $\mathrm{d} 18: 1, \mathrm{~d} 18: 2$ and $\mathrm{d} 18: 3 \mathrm{were} \mathrm{m} / \mathrm{z} 264.3$, $\mathrm{m} / \mathrm{z} 262.3$ and $\mathrm{m} / \mathrm{z}$ 260.2, respectively [14-16]. In the case of glucosylceramide molecules 19 consisting of $\mathrm{t} 18: 1$, the loss of glucose $[\mathrm{M}+\mathrm{H}-162]^{+}$was used as the precursor ion and the 20 product ions corresponding to $\mathrm{t} 18: 1$ were $\mathrm{m} / \mathrm{z} 280.3$ and $\mathrm{m} / \mathrm{z} 262.3$ [16]. Pairs of the

21 structurally specific product ions of sphingoid bases and their precursor ions were used for $\tau$ 22 the identification of glucosylceramide molecules.

\section{Results and Discussion}


1 In the positive full scan mode, $[\mathrm{M}+\mathrm{Na}]^{+},[\mathrm{M}+\mathrm{H}]^{+}$and $\left[\mathrm{M}+\mathrm{H}-\mathrm{H}_{2} \mathrm{O}\right]^{+}$were the predominant

2 signals in each peak. It is well known that the sugar moiety of glycosylceramides in plants is

3 mostly glucose [22]. In the case of molecules consisting of t18:1, the loss of glucose

$4 \quad[\mathrm{M}+\mathrm{H}-162]^{+}$was also clearly detected. Glucosylceramide molecules containing d18:2 and

5 t18:1 were determined both in maize and in rice as described previously (Fig. 1A and 2A) [16].

6 In the case of rice glucosylceramide, molecules consisting of d18:1 were also identified.

7 Detection of glucosylceramide consisting of d18:2 and t18:1 was separated into two peaks, cis-

8 and trans- isomers of $\Delta 8$-unsaturated sphingoid bases. Cis-isomer was detected earlier than

9 trans-isomer by separation of reverse phase [20]. Predominantly hydroxy fatty acids containing 16 to 26 carbon atoms were detected both in maize and in rice glucosylceramides.

We verified that the characteristic product ion at $\mathrm{m} / \mathrm{z} 260.2$ corresponding to $\mathrm{d} 18: 3$ was detected in maize glucosylceramide using the auto MS/MS detection mode (Fig. 1A). Five peaks in the total ion chromatogram of maize glucosylceramide showed the product ion at $\mathrm{m} / \mathrm{z}$

14260.2 (peak 1-5 in Fig. 1A). The MS spectra of those 5 peaks are shown in Figure 1B-F. As the precursor ion of $\mathrm{m} / \mathrm{z} 260.2,[\mathrm{M}+\mathrm{H}-18]^{+}$ions at $\mathrm{m} / \mathrm{z} 694.5,722.5,750.6,778.6$ and 806.6 were detected. The identification of each peak component is summarized in Table 1. The acylated

17 fatty acid moieties were hydroxy fatty acids with 16-24 carbon atoms. In the case of rice, glucosylceramide consisted of d18:3-C18:0h and d18:3-C20:0h (Fig. 2 and Table 1).

It has been reported that the sphingoid bases in marine invertebrates are quite different from those in mammals and in plants [13]. Triene bases with conjugated diene, such as

21 2-amino-4,8,10-octatriene-1,3-diol (d18:3) and 2-amino-9-methyl-4,8,10-octatriene-1,3-diol 22 (d19:3), were identified in marine invertebrates including ascidians [17], starfish [18, 19] and 23 squid [23] and also some fungi [24]. We have also reported that sea cucumber 
1 glucosylceramide has sphingoid bases with three double bonds [25]. . Sperling et al.

2 described the presence of sphingatrienine in tobacco leaf by HPLC analysis of sphingoid base

3 derivatized with dinitrophenyl [20]. In this study, we identified several molecular species of

4 sphingatrienine-containing glucosylceramides in maize and rice by LC-MS/MS system.

5 However, the locations of double bonds in sphingatrienine structure have not been identified.

6 Sphingolipids of plant organisms contain primarily $\mathrm{d} 18: 1^{8}, \mathrm{~d} 18: 2^{4,8}$ and $\mathrm{t} 18: 1^{8}$ as sphingoid bases

$7 \quad$ andsphingolipid $\Delta 4$-desaturase and sphingolipid $\Delta 8$-desaturase have been identified in plants [22].

8 It has been reported that the composition of sphingoid bases differs between chilling sensitive

9 and tolerant plants [26]. Details of tissue distribution, synthetic pathways and functions of plant sphingatrienines remain to be elucidated.

Recently, dietary sphingolipids have gained attention for their potential to protect the intestine from inflammation and cancer [4-9]. We reported that the daily intake of plant-origin glucosylceramides in Japan is estimated to be $50 \mathrm{mg}$ due to their presence in foodstuffs [27] and

14 we investigated the digestion and absorption of plant-derived sphingolipids [21]. Our findings indicate that the metabolic fate of plant-derived sphingoid bases, such as 4,8-sphingadienine, within enterocytes differs from that of sphingosine. Sphingoid bases, except for sphingosine, appear to be transported out of cells across the apical membranes of enterocytes by P-glycoprotein after absorption and consequently the intestinal uptake is quite poor [28, 29]. Thus, the determination of sphingolipid structures, including variation of the sphingoid backbone, from dietary sources is important to understand their functional and nutritional significance.

In this study, we analyzed the chemical structures of glucosylceramides from maize and 22 from rice using liquid chromatography-ion trap mass spectrometry. Our results indicate the 23 presence of sphingatrienine (d18:3) in higher plant sphingolipids. MS/MS analysis is a 
1 powerful method to identify the molecular structures of sphingolipids from biological sources.

2

3

4 Acknowledgement This work was supported by the Program for Promotion and Applied

5 Researches for Innovations in Bio-oriented Industry (BRAIN).

6 
1

\section{References}

1. Spiegel S, Merrill AH Jr. (1996) Sphingolipid metabolism and cell growth regulation. FASEB J 10: 1388-1397

2. Cuvillier O (2002) Sphingosine in apoptosis signaling. Biochim Biophys Acta 1585: $153-162$

3. Hannun YA, Obeid LM (2008) Principles of bioactive lipid signaling: lessons from sphingolipids. Nat Rev Mol Cell Biol 9: 139-150

4. Duan RD, Nilsson $\AA$ (2009) Metabolism of sphingolipids in the gut and its relation to inflammation and cancer development. Prog Lipid Res 4: 62-72

5. Schmelz EM (2004) Sphingolipids in the chemoprevention of colon cancer. Front Biosci 9: 2632-2639

6. Schmelz EM, Sullards MC, Dillehay DL, Merrill AH Jr. (2000) Colonic cell proliferation and aberrant crypt formation are inhibited by dietary glycosphingolipids in 1,2-dimethylhydrazine-treated CF1 mice. J Nutr 130: 522-527

7. Schmelz EM, Roberts PC, Kustin EM, Lemonnier LA, Sullards MC, Dillehay DL, Merrill AH Jr. (2001) Modulation of intracellular $\beta$-catenin localization and intestinal tumorigenesis in vivo and in vitro by sphingolipids. Cancer Res 61: 6723-6729

8. Aida K, Kinoshita M, Tanji M, Sugawara T, Tamura M, Ono J, Ueno N, Ohnishi M (2005) Prevention of aberrant crypt foci formation by dietary maize and yeast cerebrosides in 1,2-dimethylhydrazine-treated mice. J Oleo Sci 54: 45-49

9. Kinoshita M, Aida K, Tokuji Y, Sugawara T, Ohnishi M (2009) Effects of dietary plant cerebroside on gene expression in the large intestine of 1,2-dimethylhydrazine (DMH)-treated mice determined by DNA microarray analysis. J Food Lipids 16: 200-208 
10. Tsuji K, Mitsutake S, Ishikawa J, Takagi Y, Akiyama M, Shimizu H, Tomiyama T, Igarashi Y (2006) Dietary glucosylceramide improves skin barrier function in hairless mice. J Dermatol Sci 44: 101-107

11. Duivenvoorden I, Voshol PJ, Rensen PC, van Duyvenvoorde W, Romijn JA, Emeis JJ, Havekes LM, Nieuwenhuizen WF (2006) Dietary sphingolipids lower plasma cholesterol and triacylglycerol and prevent liver steatosis in APOE*3Leiden mice. Am J Clin Nutr 84: $312-321$

12. Kinoshita M, Hori N, Aida K, Sugawara T, Ohnishi M (2007) Prevention of melanin formation by yeast cerebroside in B16 mouse melanoma cells. J Oleo Sci 56: 645-648

13. Sperling P, Heinz E (2003) Plant sphingolipids: structural diversity, biosynthesis, first gene and functions. Biochim Biophys Acta 1632: 1-15

14. Bartke N, Fischbeck A, Humpf HU (2006) Analysis of sphingolipids in potatoes (Solanum tuberosum L.) and sweet potatoes (Ipomoea batatas (L.) Lam.) by reversed phase high-performance liquid chromatography electrospray ionization tandem mass spectrometry (HPLC-ESI-MS/MS). Mol Nutr Food Res 50: 1201-1211

15. Shaner RL, Allegood JC, Park H, Wang E, Kelly S, Haynes CA, Sullards MC, Merrill AH Jr. (2009) Quantitative analysis of sphingolipids for lipidomics using triple quadrupole and quadrupole linear ion trap mass spectrometers. J Lipid Res 50: 1692-1707

16. Sugawara T, Aida K, Duan J, Hirata T (2010) Analysis of glucosylceramides from various sources by liquid chromatography-ion trap mass spectrometry. J Oleo Sci in press

17. Duran R, Zubia E, Ortega MJ, Naranjo S, Salva J (1998) Phallusides, new glucosphingolipids from the ascidian Phallusia fumigate. Tetrahedron 54: 14597-14602

18. Jin W, Rinehart KL, Jares-Erijman EA (1994) Ophidiacerebrosides: cytotoxic 
glycosphingolipids containing a novel sphingosine from a sea star. J Org Chem 59: 144-147

19. Diaz de Vivar ME, Seldes AM, Maier MS (2002) Two novel glucosylceramides from gonads and body walls of the Patagonian starfish Allostichaster inaequalis. Lipids 37: 597-603

20. Sperling P, Franke S, Lüthje S, Heinz E (2005) Are glucocerebrosides the predominant sphingolipids in plant plasma membranes? Plant Physiol Biochem 43: 1031-1038

21. Sugawara T, Kinoshita M, Ohnishi M, Nagata J, Saito M (2003) Digestion of maize sphingolipids in rats and uptake of sphingadienine by Caco-2 cells. J Nutr 133: 2777-2782

22. Warnecke D, Heinz E (2003) Recently discovered function of glucosylceramides in plants and fungi. Cell Mol Life Sci 60: 919-941

23. Ohashi Y, Tanaka T, Akashi S, Morimoto S, Kishimoto Y, Nagai Y (2000) Squid nerve sphingomyelin containing an unusual sphingoid base. J Lipid Res 41: 1118-1124

24. Shu RG, Wang FW, Yang YM, Liu YX, Tan RX (2004) Antibacterial and xanthine oxidase inhibitory cerebrosides form Fusarium sp. IFB-121, an endophytic fungus in Quercus variabilis. Lipids 39: 667-673

25. Sugawara T, Zaima N, Yamamoto A, Sakai S, Noguchi R, Hirata T (2006) Isolation of sphingoid bases of sea cucumber cerebrosides and their cytotoxicity against human colon cancer cells. Biosci Biotechnol Biochem 70: 2906-2912

26. Imai, H, Ohnishi M, Hotsubo K, Kojima M, Ito S (1997) Sphingoid base composition of cerebrosides from plant leaves. Biosci Biotechnol Biochem 61: 351-353

27. Sugawara T, Miyazawa T (1999) Separation and determination of glycolipids from edible plant sources by high-performance liquid chromatography and evaporative light-scattering detection. Lipids 34: 1231-1237

28. Sugawara T, Kinoshita M, Ohnishi M, Tsuzuki T, Miyazawa T, Nagata J, Hirata T, Saito M 
(2004) Efflux of sphingoid bases by P-glycoprotein in human intestinal Caco-2 cells. Biosci

Biotechnol Biochem 68: 2541-2546

29. Sugawara T, Tsuduki T, Yano S, Hirose M, Duan J, Aida K, Ikeda I, Hirata T (2010) Intestinal absorption of dietary maize glucosylceramide in lymphatic duct cannulated rats. $\mathbf{J}$ Lipid Res in press 
1 Table 1 Glucosylceramides containing sphingatrienine (d18:3) from maize and from rice

2 identified by HPLC-MS/MS analysis.

3

\begin{tabular}{cccccc}
\hline Peak No. in & Precursor ion $\mathrm{m} / \mathrm{z}$ & Product ion & Molecule & Source \\
\cline { 2 - 4 } Fig. 1 and 2 & {$[\mathrm{M}+\mathrm{H}]^{+}$} & {$[\mathrm{M}+\mathrm{H}-18]^{+}$} & $\mathrm{m} / \mathrm{z}$ & \\
\hline 1 & 712.5 & 694.5 & 260.2 & $\mathrm{~d} 18: 3-\mathrm{C} 16: 0 \mathrm{~h}$ & Maize \\
2 & 740.5 & 724.5 & 260.2 & $\mathrm{~d} 18: 3-\mathrm{C} 18: 0 \mathrm{~h}$ & Maize, Rice \\
3 & 768.6 & 750.6 & 260.2 & $\mathrm{~d} 18: 3-\mathrm{C} 20: 0 \mathrm{~h}$ & Maize, Rice \\
5 & 796.6 & 778.6 & 260.2 & $\mathrm{~d} 18: 3-\mathrm{C} 22: 0 \mathrm{~h}$ & Maize \\
\hline
\end{tabular}

4 


\section{$1 \quad$ Figure Legends}

2

3 FIG. 1. Total ion and selected ion chromatograms of maize glucosylceramide (A) and mass 4 spectra of peak components (B-F).

5

6 FIG. 2. Total ion and selected ion chromatograms of rice glucosylceramide (A) and mass $7 \quad$ spectra of peak components (B and C). 

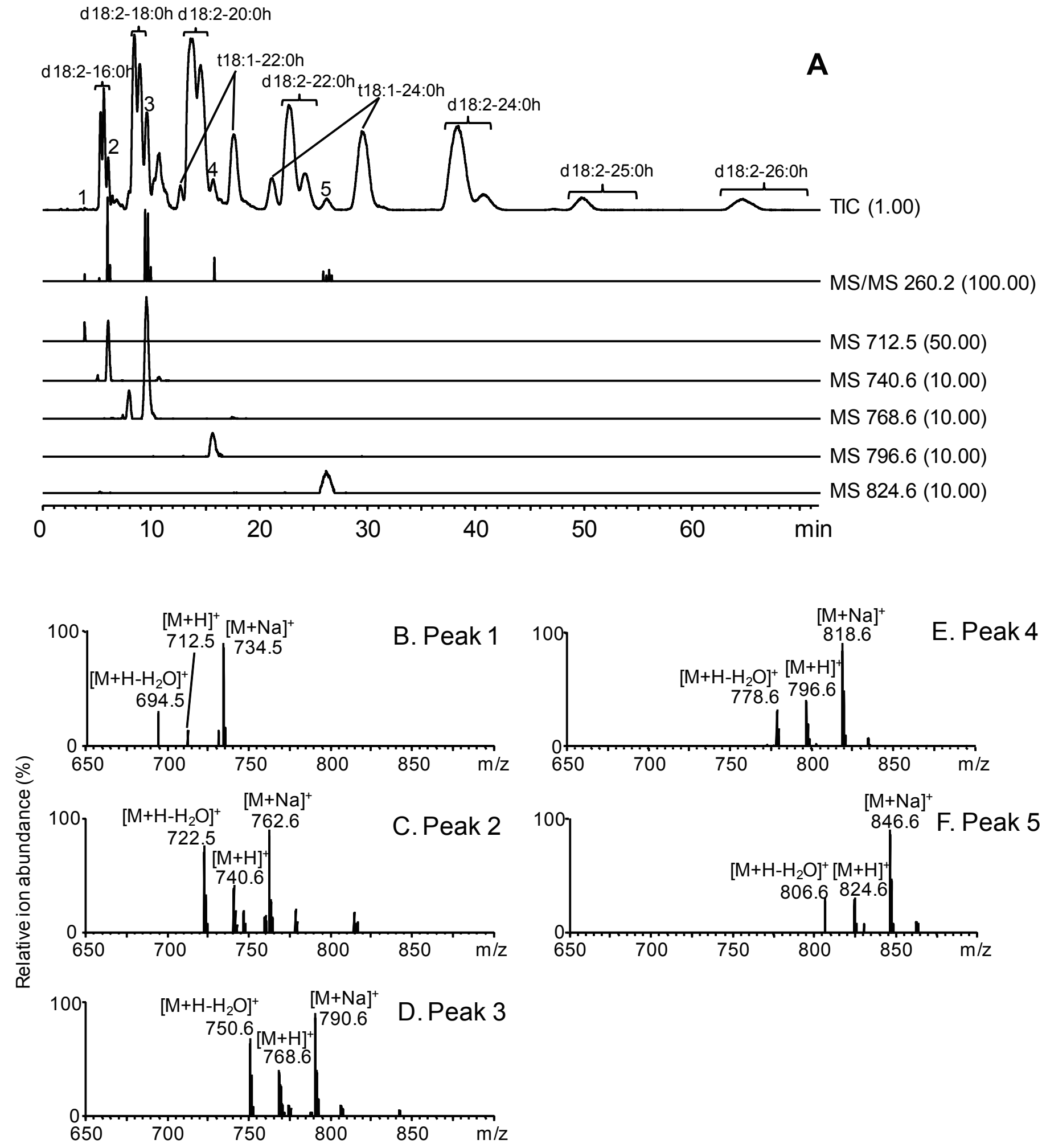

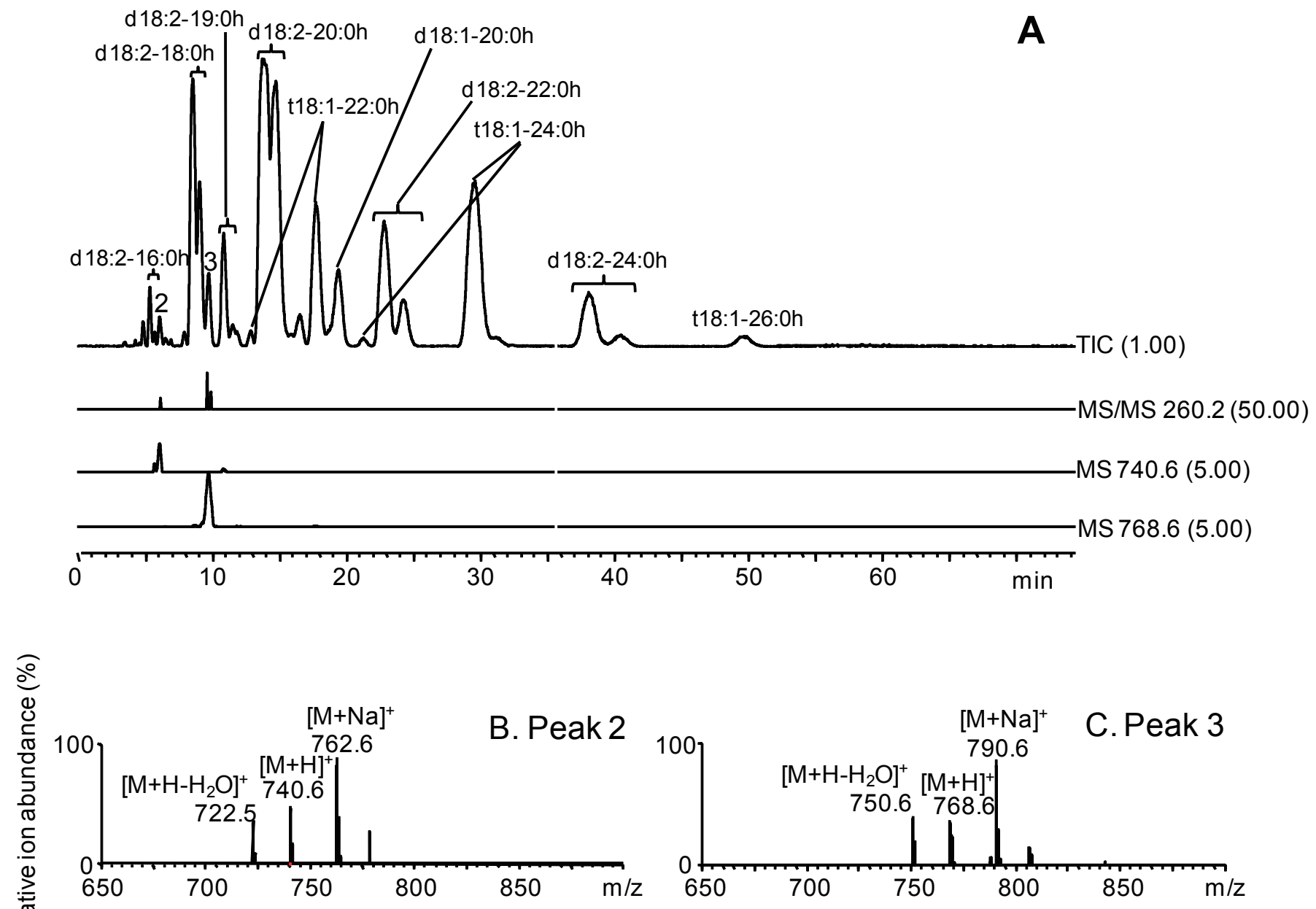